\title{
Intervention of Peiyuan Huayu Decoction on the neuron damage in model rats with acute subdural hematoma
}

\author{
Xiao-Xuan Fan ${ }^{1}$, Xiao-Ping Zhao ${ }^{1 \bowtie}$, Xin-Rong Guo ${ }^{2}$, Hai-Feng Duan ${ }^{1}$, Ge-Ting Liang ${ }^{1}$ \\ ${ }^{1}$ Neurosurgery Department, Affiliated Hospital of Shaanxi University of Chinese Medicine, Xianyang City, Shaanxi Province, 712000 \\ ${ }^{2}$ Acupuncture and Massage College, Shaanxi University of Chinese Medicine, Xianyang City, Shaanxi Province, 712046
}

\section{ARTICLE INFO}

Article history:

Received 1 May 2017

Received in revised form 12 May 2017

Accepted 20 May 2017

Available online 1 July 2017

\section{Keywords:}

Acute subdural hematoma

Peiyuan Huayu Decoction

Neuron

S100 $\beta$ protein

Neuron-specific enolase

\section{ABSTRACT}

Objective: To study the intervention effect of Peiyuan Huayu Decoction on the neuron damage in model rats with acute subdural hematoma (ASDH). Methods: 160 SD rats were randomly divided into four groups, and the ASDH model rats were made by stereotactic autoblood injection, and sham operation group received craniotomy without blood injection. Sham operation group and model group were normally bred after model establishment, and $6 \mathrm{~h}$ after model establishment, the treatment group received intragastric administration of Peiyuan Huayu Decoction, and control group received intragastric administration of Piracetam Tablets, 1 time a day. On the $1 \mathrm{~d}, 3 \mathrm{~d}, 5 \mathrm{~d}$ and $7 \mathrm{~d}$ after model establishment, the general conditions of rats (activity, food intake and mental state) were observed, blood was collected via auricula dextra, ELISA method was used to determine peripheral plasma NSE and S100 $\beta$ protein contents, routine HE staining was conducted after perfusion fixation, the neurons in blood injection side of brain tissue were counted, and the neuron damage was observed. Results: 26 rats were dead in the experiment. The general conditions of sham operation group were significantly better than those of other groups, treatment group was significantly better than model group and control group on the $5 \mathrm{~d}$ group $(P<0.05)$, and there was no significant difference on the $1 \mathrm{~d}, 3 \mathrm{~d}$ and $7 \mathrm{~d}(P>0.05)$; neuron count of sham operation group was basically stable, treatment group was not different from model group and control group on the $1 \mathrm{~d}(P>0.05)$, treatment group was better than model group $(P<0.05)$, and not different from control group $(P>0.05)$ on the $3 \mathrm{~d}$, and treatment group was better than model group and control group on the $5 \mathrm{~d}$ and $7 \mathrm{~d}$ $(P<0.05)$; peripheral plasma S100 $\beta$ protein and NSE contents of sham operation group were at lower levels, treatment group was not significantly different from model group and control group on the $1 \mathrm{~d}(P>0.05), \mathrm{S} 100 \beta$ protein and NSE contents decreased significantly on the $3 \mathrm{~d}$, and treatment group was significantly different from model group and control group $(P<0.05)$, S100 $\beta$ protein and NSE contents increased on the $5 \mathrm{~d}$ and $7 \mathrm{~d}$, the increase in treatment group was slower than that in model group and control group, and there was significant difference $(P<0.05)$. Conclusion: Peiyuan Huayu Decoction has obvious protective effect on the neurons in ASDH model rats, and this effect may be based on the inhibition of secondary neuron damage.

\section{Introducion}

Acute subdural hematoma (ASDH) is a common neurosurgical disease when craniocerebral injury leads to acute intracranial hemorrhage and the blood gathers in subdural space [1,2], which is often accompanied by parenchymal damage or complicated by other intracranial hematoma. Clinical application of Peiyuan Huayu Decoction has obvious therapeutic effect for ASDH, this experiment

\footnotetext{
${ }^{\square}$ Corresponding author: Xiao-Ping Zhao, Neurosurgery Department, Affiliated Hospital of Shaanxi University of Chinese Medicine, Xianyang City, Shaanxi Province, 712000 .

Tel: 13992092318

E-mail: zxp9918@sina.com
}

was carried out in order to observe the intervention effect of the decoction on neuron damage in ASDH model rats, and the content was as follows.

\section{Materials and methods}

\subsection{Experimental animals and groups}

8 week old healthy clean SD rats with body weight $(260 \pm 25) \mathrm{g}$ were selected, half male and half female (number SCXK: 20120007), and the 160 rats were randomly divided into 4 groups, 40 in each group. 


\subsection{Model making methods}

Model making methods of Wang $\mathrm{J}$ and others [3] were referred, the SD rats were given intraperitoneal injection of $3.5 \%$ chloral hydrate for anesthesia, the head was fixed in stereotactic apparatus after anesthesia, routine skin preparation and disinfection were conducted, a top median longitudinal incision about $5 \mathrm{~mm}$ long was made, and the scalp and muscle layer were peeled to the right to expose the right coronal and sagittal suture; wedge-shaped high-speed drill was used to drill a $1 \mathrm{~mm}$-diameter bone window $3 \mathrm{~mm}$ from right coronal and sagittal suture, $1 \mathrm{ml}$ femoral venous blood was collected and injected at $50 \mu \mathrm{L} / \mathrm{s}$ in subdural space with micro syringe, and $500 \mu \mathrm{L}$ blood was injected. The needle inserting process should avoid arachnoid puncture by needle tip, the sham operation group received same operation procedure without autoblood injection, and heating blanket temperature was kept at $37{ }^{\circ} \mathrm{C}$ to maintain the body temperature of rats.

\subsection{Therapy}

Control group and model group were normally bred, the treatment group were given intragastric administration of Peiyuan Huayu Lishui Decoction 6h after model establishment (the prescription was made up of the radix astragali $30 \mathrm{~g}$, Chinese angelica $15 \mathrm{~g}$, prepared rehmannia root $12 \mathrm{~g}$, dried rehmannia root $12 \mathrm{~g}$, radix codonopsis $6 \mathrm{~g}$, safflower $12 \mathrm{~g}$, etc, and the medicines were all purchased by Pharmacy Department of Affiliated Hospital of Shaanxi University of Chinese Medicine, all crude drugs in the prescription were mixed with water in proportion of $1: 8(\mathrm{~g} / \mathrm{mL})$, soaked for 30 min before decocting, continued to be decocted for $30 \mathrm{~min}$ after boiling, and filtered; the drugs were mixed with water in proportion of $1: 6(\mathrm{~g} / \mathrm{mL})$ and decocted one more time. The filtrate from two times of decoction were combined for rotary evaporation at $60{ }^{\circ} \mathrm{C}$ and condensed into $0.6 \mathrm{~g} / \mathrm{mL}$ of soup, single dose $10 \mathrm{~mL} / \mathrm{kg}, 1$ time/day), and control group were given intragastric administration of Piracetam Tablets (Piracetam Tablets, 0.4g/tablet, produced by Yichang Humanwell Pharmaceutical Co., Ltd., batch number: 3140616 . The tablets were grinded and configured to $0.09 \mathrm{~g} / \mathrm{mL}$ suspension liquid, single dose $1.2 \mathrm{~g} / \mathrm{kg}, 1$ time/day).

\subsection{Observation indexes: observed on the $1 d, 3 d, 5 d$ and $7 d$ after model establishment}

(1) Evaluation criteria (self-made) for general status: activity (quick response: 4 points, a bit slow: 3 points, slow: 2 points, poor locomotor activity: 1 point); food intake (the daily food intake $\geqq 30 \mathrm{~g}$ : 4 points, $10 \mathrm{~g} \leqq$ food intake $<30 \mathrm{~g}$ : 3 points, food intake $<$ $10 \mathrm{~g}$ but could eat: 2 points, no autonomous food intake: 1 point); mental state (normal: 4 points, poor: 3 points, dispirited: 2 points, lethargic: 1 point).

(2) Neuron count: infusion needle was directly inserted into the left ventricle, infusion apparatus was opened and velocity was observed, auricula dextra was quickly cut open, blood flowed out, $200 \mathrm{ml}$ saline was injected, about $200 \mathrm{ml} 4 \%$ paraformaldehyde was injected rapidly after lung tissue turned white, the dripping speed was showed down after the rat tail tilted, and $250 \mathrm{ml}$ paraformaldehyde was injected at about 2 drops/sec. Injection was stopped when the
SD were completely rigid, and the head was opened to take out of the brain. Coronal-section brain tissue $2 \mathrm{~mm}$ from the front and back of injection pinhole was collected, dehydrated, fixed, paraffinembedded, serially coronally sliced and conventionally stained with HE, and the right cerebral neurons were counted under optical microscope. Six nonoverlapping photos were randomly taken in 400 times view. The Image-Pro Plus 6.0 image analysis software was used for the image analysis, and the number of living neurons in the view was counted.

(3) Plasma NSE and S100 determination:after intraperitoneal injection of $3.5 \%$ chloral hydrate for anesthesia, blood was collected from auricula dextra, and plasma NSE and S100 $\beta$ protein contents were determined by ELISA method.

\subsection{Statistical methods}

IBM SPSS Statistics 19.00 software was used for data analysis Data of all groups were in terms of $\bar{x} \pm \mathrm{s}$. Mean comparison between two groups was by t test, and $P<0.05$ indicated statistical significance in differences.

\section{Results}

The total number of dead rats in this experiment was 26 , including 0 in the sham operation group, 11 in the model group, 6 in the treatment group and 9 in the control group. A total of 134 were enrolled as observation samples.

\subsection{General condition}

The general conditions (activity, food intake and mental state) of rats after model establishment were significantly worse than those of sham operation group, on the $1 \mathrm{~d}$, the conditions of sham operation group were basically normal, the condition grading of other groups decreased significantly, there was no significant difference among model group, treatment group and control group $(P>0.05)$, the general conditions of animals increased gradually after that, the increase in treatment group was faster than that in model group and control group, the grading on the $5 \mathrm{~d}$ was significantly different from that of model group and control group $(P<0.05)$, and the grading was not significantly different among groups on the $7 \mathrm{~d}(P>0.05)$, shown in Table 1 and Figure 1.

Table 1

Comparison of general condition grading among groups

\begin{tabular}{lcccc}
\hline Groups & $1 \mathrm{~d}$ & $3 \mathrm{~d}$ & $5 \mathrm{~d}$ & $7 \mathrm{~d}$ \\
\hline Sham operation group & $11.33 \pm 0.82$ & $11.17 \pm 0.75$ & $11.5 \pm 0.55$ & $11.83 \pm 0.41$ \\
Model group & $4.16 \pm 1.17$ & $4.67 \pm 1.37$ & $8.17 \pm 1.17$ & $10.83 \pm 0.89$ \\
Treatment group & $3.83 \pm 0.98^{\triangle}$ & $6.17 \pm 1.17 *$ & $10.67 \pm 1.21^{\#}$ & $11.67 \pm 0.52 \%$ \\
Control group & $3.67 \pm 0.82$ & $4.83 \pm 1.17$ & $9.17 \pm 1.17$ & $11.17 \pm 0.75$ \\
\hline
\end{tabular}

Note: ${ }^{\triangle}$ on the $1 \mathrm{~d}$, compared with model group $(t=0.598, \mathrm{p}>0.05)$, compared with control group $(t=1.000, P>0.05)$; $*$ on $3 \mathrm{~d}$, compared with model $\operatorname{group}(t=2.236, \mathrm{p}>0.05)$, compared with control group $(t=2.000, P>0.05)$; on the $5 \mathrm{~d}$, compared with model group $(t=7.319, P<0.05)$, compared with control group $t=3.00, P<0.05) . ※$ on the $7 \mathrm{~d}$, compared with model group $(t=1.746, P>0.05)$, compared with control group $(t=1.464, P>0.05)$. 


\subsection{Neuron count}

After model establishment, the number of neurons of sham operation group was stable. On the $1 \mathrm{~d}$, the number of survival neurons of model group, treatment group and control group were significantly lower than that of sham operation group, and the number of neurons was not significantly different among model group, treatment group and control group $(P>0.05)$; on the $3 \mathrm{~d}$, the number of neurons of treatment group was significantly higher than that of model group $(P<0.05)$, and not different from that of control group $(P>0.05)$; on the $5 \mathrm{~d}$, the number of neurons of treatment group was significantly higher than that of model group and control group $(P<0.05)$, and the number of control group increased, too; on the $7 \mathrm{~d}$, the number of neurons of treatment group significantly increased, and was significantly higher than that of model group and control group $(P<0.05)$, shown in Table 2, Figure 2 and Figure 3.

Table 2

Comparison of blood injection-side neuron count among groups (n).

\begin{tabular}{lcccc}
\hline Groups & $1 \mathrm{~d}$ & $3 \mathrm{~d}$ & $5 \mathrm{~d}$ & $7 \mathrm{~d}$ \\
\hline $\begin{array}{l}\text { Sham operation } \\
\text { group }\end{array}$ & $28.50 \pm 3.27$ & $25.5 \pm 3.27$ & $24.00 \pm 3.16$ & $26.67 \pm 3.44$ \\
Model group & $15.00 \pm 3.16$ & $10.83 \pm 3.19$ & $9.17 \pm 2.79$ & $13.33 \pm 2.66$ \\
$\begin{array}{l}\text { Treatment } \\
\text { group }\end{array}$ & $16.17 \pm 3.31^{\triangle}$ & $15.67 \pm 3.01^{*}$ & $19.17 \pm 3.31^{\#}$ & $24.83 \pm 3.06^{*}$ \\
Control group & $17.17 \pm 2.64$ & $15.00 \pm 3.74$ & $16.83 \pm 2.56$ & $20.33 \pm 3.01$ \\
\hline
\end{tabular}

Note: ${ }^{\triangle}$ on the $1 \mathrm{~d}$, compared with model group $(t=1.746, P>0.05)$, compared with control group $(t=2.236, P>0.05)$; *n the $3 \mathrm{~d}$, compared with model group $(t=29.000, \mathrm{p}<0.05)$, compared with control group $(t=1.581, P>0.05)$; \#on the 5d, compared with model group ( $t=27.381, P<0.05)$, compared with

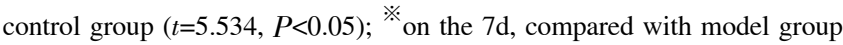
$(t=26.858, P<0.05)$, compared with control group $(t=10.510, P<0.05)$.

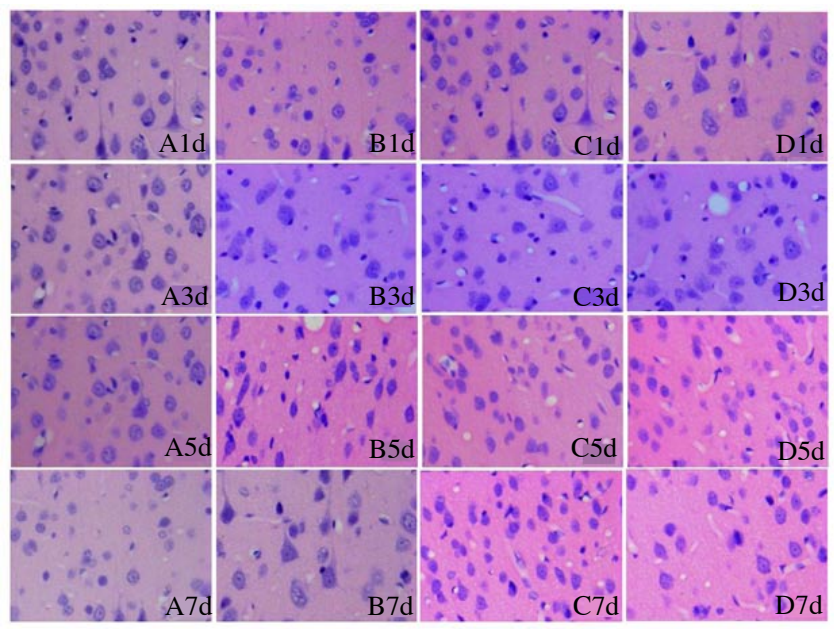

Figure 1. Routine HE staining neuron expression $(\times 400)$.

A group was for sham operation group, B group was for model group, C group was for treatment group,D group was for control group.

As shown in Figure 1, A group was for sham operation group, the number of survival neurons didn't change significantly; B group was for model group, the number of neurons continued to decrease after model establishment, and began to increase from the $7 \mathrm{~d}$; C group was for treatment group, the number of neurons decreased significantly after model establishment, the decrease level was less than that of model group and control group, and it began to increase significantly from the $5 \mathrm{~d}$; D group was for control group, the number of neurons decreased significantly after model establishment, the decrease level was less than model group and more than treatment group, and it began to increase slowly from the $5 \mathrm{~d}$.

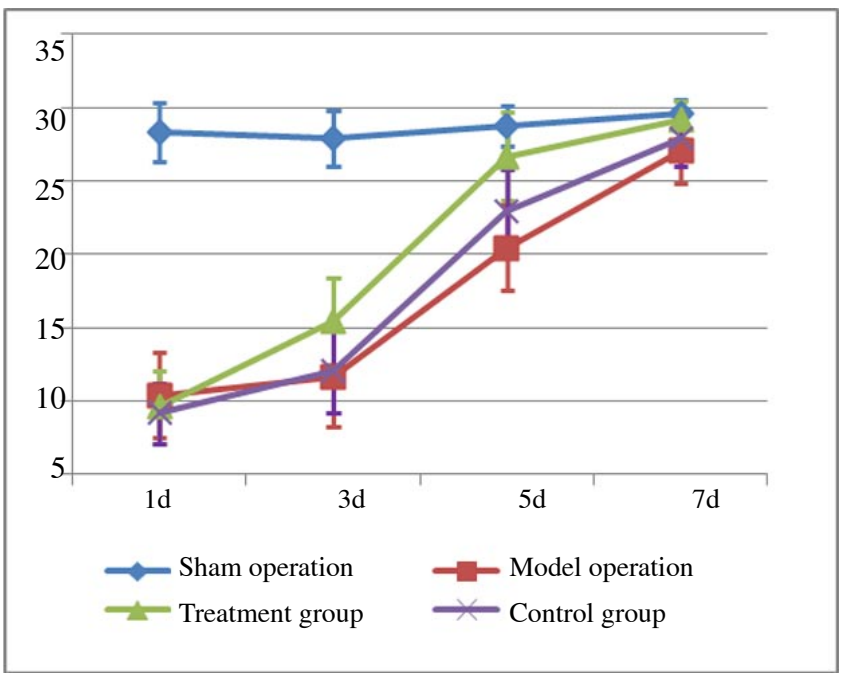

Figure 2. General condition comparision among groups.

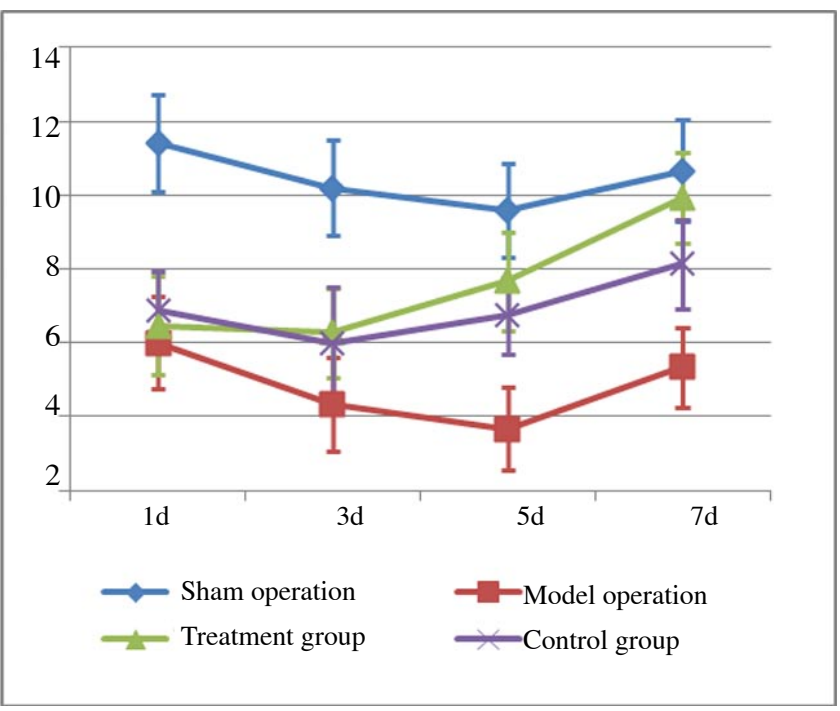

Figure 3. Neuron count comparision among groups.

\subsection{Plasma S100 $\beta$ and NSE detection}

S100 $\beta$ of sham operation group was at lower levels all the time, that of other groups significantly increased on the $1 \mathrm{~d}$ after model establishment, there was no difference among model group, treatment group and control group ( $P>0.05)$, it reduced to the lowest point on the $3 \mathrm{~d}$ and continued to increase after that; on the $3 \mathrm{~d}, \mathrm{~S} 100$ $\beta$ content of treatment group was lower than that of model group 
and control group $(P<0.05)$, and it increased after that and increased more slowly than other groups (compared with model group and control group on the $5 \mathrm{~d}$ and $7 \mathrm{~d}, P<0.05)$, shown in Table 3 and Figure 4 . The changes of NSE content were basically consistent with those of $\mathrm{S} 100 \beta$, sham operation group was at lower levels all the time, other groups increased significantly on the $1 \mathrm{~d}$ after model establishment, and there was no difference among model group, treatment group and control group $(P>0.05)$; treatment group was significantly different from model group on the $3 \mathrm{~d}(P<0.05)$, but not significantly different from that of control group $(P>0.05)$, the difference was more significant on the $5 \mathrm{~d}$ and $7 \mathrm{~d}$, and the NSE content of treatment group was significantly lower than that of model group $(P<0.05)$ and control group $(P<0.05)$, shown in Table 4 and Figure 5.

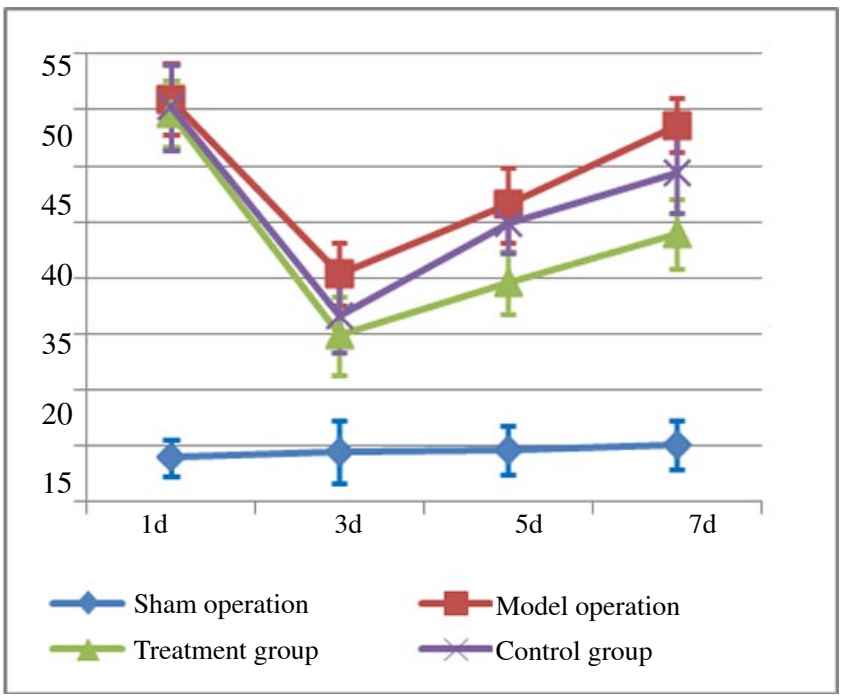

Figure 4. Plasma S100 $\beta$ content comparison among groups.

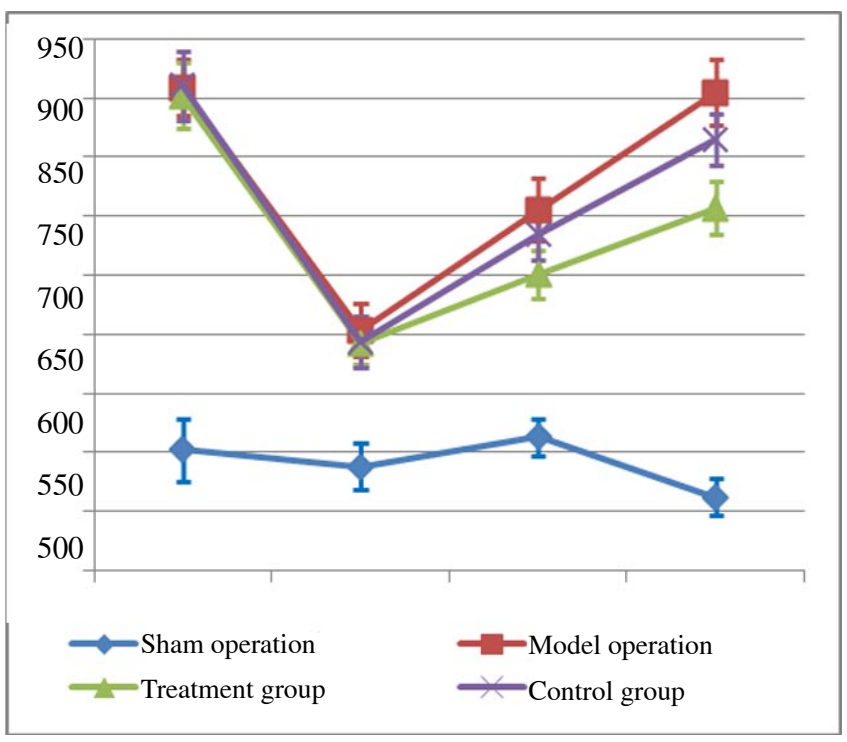

Figure 5. Plasma NSE content comparison among groups.
Table 3

Comparison of plasma S100 $\beta$ contents among groups (ng/L).

\begin{tabular}{ccccc}
\hline Groups & $1 \mathrm{~d}$ & $3 \mathrm{~d}$ & $5 \mathrm{~d}$ & $7 \mathrm{~d}$ \\
\hline $\begin{array}{c}\text { Sham } \\
\text { operation }\end{array}$ & $18.98 \pm 1.67$ & $19.50 \pm 2.81$ & $19.63 \pm 2.15$ & $20.07 \pm 2.18$ \\
group & & & & \\
Model & & & & \\
group & $50.98 \pm 3.22$ & $35.38 \pm 2.75$ & $41.58 \pm 3.34$ & $48.65 \pm 2.46$ \\
Treatment & $49.70 \pm 2.96^{\triangle}$ & $29.87 \pm 3.52 *$ & $34.57 \pm 2.77^{\#}$ & $38.95 \pm 3.06^{*}$ \\
group & & & & \\
Control \\
group & $50.23 \pm 3.77$ & $31.57 \pm 3.18$ & $39.83 \pm 2.54$ & $44.4 \pm 3.57$
\end{tabular}

Note: ${ }^{\triangle}$ on the $1 \mathrm{~d}$, compared with model group $(t=1.433, P>0.05)$, compared with control group $(t=0.890, P>0.05$; *on the $3 \mathrm{~d}$, compared with model group $(t=9.494, P<0.05)$, compared with control group $(t=6.336, P<0.05)$; *\#on the $5 \mathrm{~d}$, compared with model group $(t=16.938, P<0.05)$, compared with control group $(t=18.05$, $P<0.05) ; * *$ on the $7 \mathrm{~d}$, compared with model group $(t=31.638$, $P<0.05)$, compared with control group $(t=16.060, P<0.05)$.

Table 4

Comparison of plasma NSE contents among groups $(\mu \mathrm{g} / \mathrm{L})$

\begin{tabular}{|c|c|c|c|c|}
\hline Groups & $1 d$ & $3 d$ & $5 d$ & $7 d$ \\
\hline Sham & & & & \\
\hline $\begin{array}{c}\text { operation } \\
\text { group }\end{array}$ & $602.5 \pm 26.51$ & $588.2 \pm 20.17$ & $613.2 \pm 15.63$ & $562.2 \pm 15.66$ \\
\hline $\begin{array}{l}\text { Model } \\
\text { group }\end{array}$ & $908.8 \pm 23.38$ & $703.3 \pm 22.52$ & $805.3 \pm 26.36$ & $904.5 \pm 27.79$ \\
\hline $\begin{array}{c}\text { Treatment } \\
\text { group }\end{array}$ & $901.8 \pm 27.43^{\triangle}$ & $691.5 \pm 17.19 *$ & $751.0 \pm 20.62^{\#}$ & $806.8 \pm 22.69^{*}$ \\
\hline $\begin{array}{c}\text { Control } \\
\text { group }\end{array}$ & $910.8 \pm 29.21$ & $693.3 \pm 21.63$ & $784.7 \pm 21.39$ & $865.2 \pm 21.82$ \\
\hline
\end{tabular}

Note: ${ }^{\triangle}$ on the $1 \mathrm{~d}$, compared with model group $(t=2.085, P>0.05)$, compared with control group $(t=2.293, P>0.05)$; on the $3 \mathrm{~d}$, compared with model group $(t=5.277, P<0.05)$, compared with control group $(t=0.807, P>0.05)$; \#on the $5 \mathrm{~d}$, compared with model group ( $t=20.768, P<0.05)$, compared with control group $(t=34.047$, $P<0.05) ;{ }^{*}$ on the $7 \mathrm{~d}$, compared with model group $(t=16.359$, $P<0.05)$, compared with control group $(\mathrm{t}=10.812, P<0.05)$.

\section{Discussion}

ASDH is a common intracranial space-occupying lesion after acute craniocerebral injury, it usually appears with brain contusion,brain hematoma and others at the same time, only $30-40 \%$ of the pure ASDH requires surgical treatment [4,5], and non-surgical treatment will still be a feasible method for some patients [6], such as children or the elderly who have ASDH caused by minor damage, or the ASDH with quick restoration of consciousness after injury [7]. Although the ASDH after acute craniocerebral injury often shows the increased intracranial pressure-associated pathologic changes [8-11], 
the application of decompressive craniectomy or not is not much associated with the long-term morbidity [12]. Therefore, in addition to the increased intracranial pressure, the key factors of ASDH to cause disability are also associated with the secondary neuron damage caused by increased intracranial pressure, hematoma diffusion and other processes, and reducing ASDH-induced secondary neuron damage is an effective way of treatment. Peiyuan Huayu Decoction is the empirical formula of the authors, the clinical application effect is ideal, and it is not clear whether it has exact protective effect on the neuron damage after ASDH

In this research, stereotactic $500 \mu \mathrm{L}$ autoblood injection was used to make SD rats into ASDH models, animal mortality was low, the stability of the model was good, it better simulated the pathological process of secondary injury produced by fast intracranial pressure increase and hematoma diffusion, and the model was ideal.

The general conditions (activity, food intake and mental state) of rats after model establishment were significantly worse than those of sham operation group, on the $1 \mathrm{~d}$, the status of sham operation group was basically normal, the status score of other groups significantly decreased, there was no significant difference among model group, treatment group and control group $(P>0.05)$, general status score of animals gradually increased after that, the increase of treatment group was faster than that of model group and control group, the score on the $5 \mathrm{~d}$ was significantly different from that of model group and control group $(P<0.05)$, the score was not obviously different among groups on the $7 \mathrm{~d}(P>0.05)$, it indicates that the decoction has improving effect on the general conditions of rats, and it is consistent with the clinical curative effect.

Neuron count showed that $1 \mathrm{~d}$ after model establishment, the number of survival neurons of model group, treatment group and control group were significantly lower than that of sham operation group, the neuron damage was significant after model establishment, it was in line with the clinical pathological process, and it suggested successful model establishment; the number of neurons continued to fall on the $3 \mathrm{~d}$, but the number of damaged neurons of treatment group was significantly less than that of model group $(P<0.05)$, it suggested the secondary neuron damage continued to occur, and the secondary injury is relieved after intervention. the number of neurons of treatment group increased on the $5 \mathrm{~d}$, was obviously more than that of model group and the control group $(P>0.05)$, and was more than at on 1d after model establishment, the number of control group also increased, but was still lower than that on the $1 \mathrm{~d}$ after model establishment; on the $7 \mathrm{~d}$, the number of neurons in each group increased, the number of neurons of treatment group was significantly higher than that of the model group and the control group $(P<0.05)$, and the protective effect on neurons was significant.
The number of neurons in treatment group and control group reduced to the lowest point on the $3 \mathrm{~d}$ and began to rise after that, it showed that the secondary neuron damage was slowed down, the repair began, and this was consistent with the general performance of the rats; the increase in the number of neurons of treatment group was significant, the increase of control group was slow, it indicated that the decoction had protective effect on neurons, and this effect may be based on inhibiting the secondary neuron injury.

NSE accounts for $1.5 \%$ of the total soluble proteins in the skull. Its contents range from high to low in the brain, spinal cord and the surrounding ganglion, the latter's NSE levels are far below the intracranial level by 10-100 times. Under normal circumstances, NSE exists only in neuron cytoplasm, NSE is "leaked" from the damaged neurons after craniocerebral injury, crosses through the damaged blood brain barrier and enters into the systemic circulation, and thus NSE concentration increases significantly in peripheral blood. S100 $\beta$ protein has high specificity to the glial cells and schwann cells within central nervous system, $\mathrm{S} 100 \beta$ content is very low in other tissues, it is mainly distributed in the astrocytes, schwann cells, some neurons and so on in the brain, S100 protein molecule cannot cross through the blood brain barrier under normal situation, but brain injury causes the destruction of brain cells and blood brain barrier after craniocerebral injury, blood $\mathrm{S} 100 \beta$ protein rises rapidly, blood brain barrier is further destructed if there is secondary injury, glial cell damage causes S100 $\beta$ protein spillover, its content change is closely related to the clinical symptoms, signs and imaging changes, and it is a good indicator to judge the severity of brain injury [13-15].

In this study, $\mathrm{S} 100 \beta$ had been in a low level in sham operation group, it increased obviously in the other groups after model establishment, it reduced to the lowest point on the $3 \mathrm{~d}$, and then it continued to increase; after the $3 \mathrm{~d}$, the increase of $\mathrm{S} 100 \beta$ content in treatment group was slower than that in model group and control group, and this trend was more obvious with the extension of time (compared with the $5 \mathrm{~d}$ and $7 \mathrm{~d}, P<0.05$ ). NSE content changes were basically consistent with those of $\mathrm{S} 100 \beta$, the treatment group was significantly different from model group on the $3 \mathrm{~d}(P<0.05)$, but not obviously different from control group $(P>0.05)$, the difference was more apparent on the $5 \mathrm{~d}$ and $7 \mathrm{~d}$, and the content of treatment group was significantly lower than that of model group $(P<0.05)$ and control group $(P<0.05)$. The increase of these two indicators on the 1d was mainly caused by direct neuron damage, and the increase after the $3 \mathrm{~d}$ was mainly associated with the secondary injury of neurons. Research results showed that after intervention, the increase of these two indexes was slow after the $3 \mathrm{~d}$, it indicates that neuron damage and blood brain barrier permeability are lower, the neuronal 
protective effect is significant, and this is consistent with neuron count results

Above all, Peiyuan Huayu Decoction has obvious neuron protection effect on ASDH rat model, and this effect may be based on the inhibition of secondary neuron injury.

\section{Declare of interest statement}

We declare that we have no conflict of interest.

\section{References}

[1] Lee CH, Kang DH, Hwang SH, Park IS, Jung JM, Han JW. Spontaneous rapid reduction of a large acute subdura hematoma. J Korean Med Sei 2009; 24: 1224-1226.

[2] Baeeh Li H, Behzad M, Sehleekenberger M, Buchholz HG, Heimann A, Kempski O, et al. Blood constituents trigger brain swelling, tissue death, and reduction of glucose metabolism early after acute subdural hematoma in rats. J Cereb Blood Flow Metab 2010; 30: 558-576.

[3] Wang J, Long ZJ, Ren ZX, Lu SX, Gao HW, Tian LN. Stability of acute subdural hematoma in rats developed by subdural injection of different volumes of autologous blood. Chin J Comp Med 2015; 25(9): 45-50

[4] Massaro F, Lanotte M, Faccani G, Triolo C. One hundred and twenty-seven cases of acute subdural haematoma operated on. Correlation between CT scan findings and outcome. Acto Neurochir (Wien) 1996; 138(2): 185-191.

[5] Servadei F, Nasi MT, Giuliani G, Cremonini AM, Cenni P, Zappi $\mathrm{D}$, et al. CT prognostic factors in acute subdural haematomas: the value of the 'worst' CT scan. Br J Neurosurg 2000; 14(2): 110116
[6] Vega RA, Valadka AB. Natural History of Acute Subdural Hematoma. Neurosurg Clin N Am 2017; 28(2): 247-255. doi: 10.1016/j.nec.2016.11.007. Epub 2017 Jan 30.

[7] Gan Q, Zhao H, Zhang H, You C. Rapid Spontaneously Resolving Acute Subdural Hematoma. J Craniofac Surg 2017; 28(3): e287-e289. doi: 10.1097/SCS.0000000000003533.

[8] Udoh DO. Bilateral post-traumatic acute extradural hematomas: a reportof four cases and review of literature. Niger J Clin Pract 2012; 15(1): 104-107.

[9] Le TH, Gean AD. Neuroimaging of traumatic brain injury. Mt Sinai J Med 2009; 76(2): 145-162.

[10]Zammit C, Knight WA. Severe traumatic brain injury in adults. Emerg Med Pract 2013; 15(3): 1-28.

[11]Gabrielian L, Helps SC, Thornton E, Turner RJ, Leonard AV, Vink R. Substance P antagonists as a novel intervention for brain edema and raised intracranial pressure. Acta Neurochir Suppl 2013; 118: 201-204.

[12]Phan K, Moore JM, Griessenauer C, Dmytriw AA, Scherman DB, Sheik-Ali S, et al. Craniotomy Versus Decompressive Craniectomy for Acute Subdural Hematoma: Systematic Review and Meta-Analysis. World Neurosurg. 2017 Mar 16. pii: S18788750(17)30336-4. doi:10.1016/j.wneu.2017.03.024.

[13]Wolf H, Frantal S, Pajenda GS, Salameh O, Widhalm H, Hajdu $\mathrm{S}$, et al. Predictive value of neuromarkers supported by a set of clinical criteria in patients with mild traumatic brain injury: S100 $\beta$ protein and neuron-specific enolase on trial. Neurosurg 2013; 118( 6): 1298-1303.

[14]Lange RT, Brubacher JR, Iverson GL, Procyshyn RM, Mitrovic S. Differential effects of alcohol intoxication on S100B levels following traumatic brain injury. Trauma 2010; 68(5): 1065-1071. [15]ZHANG Jiang-bo, SHU Han-sheng. The research advances of neuron-specific enolase in nerve injury. Chinese Journal of general practice 2016 ; 14(1): 125-127. 